\title{
MIDPI sciforum
}

MOL2NET, International Conference Series on Multidisciplinary Sciences Insert the title of the workshop here

Obtaining a computer-assisted QSAR model for the prediction of anti-inflammatory activity

Author $1^{a}$, Author $2^{b}$, ... etc.

${ }^{a}$ Insert affiliation here

${ }^{b}$ Insert affiliation here

\section{Graphical Abstract}

QSAR

Model

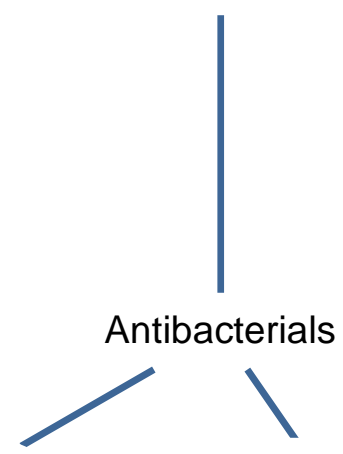

molecular descriptors
Adverse Reactions

\section{Abstract.}

Due to the high interest that represents the study from the adverse reactions to the medications and the importance of foreseeing the same ones the employment of the methods of molecular modeling with such an becomes a novel fact. In this work was carried out the calculation of the spectral moments of the adjacency matrix between edges of the molecular graph with suppressed hydrogens, pondered in the main diagonal with different parameters that characterize as much to the connections as to the atoms in the molecules of 63 compound of antibacterial action, using for it the methodology MODESLAB. 91 descriptors were calculated, which were used in a series of training divided in four groups, according to the type of adverse reaction but it frequents. With the objective of identifying the descriptors that better they discriminant against among those compound of each group and to define the group of functions of these descriptors able to distinguish with the biggest precision possible to the members of one or another group, an discriminant analysis was developed by means of the statistical software Statistical 8.5. three functions were 
generated that they constitute lineal combinations of 6 molecular descriptors, which code so much information steric as electronic of the molecules of each group. The obtained functions present a Lambda of very low minimum Wilks $(0,07)$ and a high canonical correlation $(0,82)$, that which demonstrates their power discriminant.

\section{Introduction}

The adverse reactions taken place by medications (or RAM according to their initials) they don't constitute a novelty. The origin of the concern for the consequences of the use of the medications is as old as the own existence of the drugs. Any substance with activity pharmacological potential, it can act as a remedy, but also as a poison. (Edwards, 2000)

The RAM is considered one of the main problems of the therapy with drugs. This reactions associate to factors like the morbility, the mortality, the decrease of the success of a certain, and direct therapy or indirectly, at a great medical cost. (Schlienger, 2000; Lazarou, 1998; Pirmohamed, 1998)

The employment of computer techniques for the prediction of the RAM constitutes a novel advance. If it is considered that an adverse reaction depends on the structure of a fármaco and that, also, it is a property of the same one, methods of molecular modeling could be applied. These methods would allow the obtaining of mathematical models that you/they can predict the occurrence of such adverse reactions. The setting in practice of the methodology MODESLAB in studies QSPR and QSAR have demonstrated their effectiveness. It is for it that we will serve ourselves as this methodology to calculate the molecular describers and to use them in the obtaining of the model predictives in the present investigation.

\section{Materials and Methods}

. The adverse reactions that produce the compounds employees settled down keeping in mind the approaches that it registers the Dictionary of Pharmaceutical Specialties 2017.

Descriptors. The SMILES derived from ACD/Labs were introduced in the software package MODESLAB version $1.5,{ }^{13}$ which includes the TOPS-MODE approach used to generate the molecular descriptors. The mathematical details of the method have been largely reported ${ }^{14-16}$. As a result, a matrix containing the spectral moments from $\mu_{1}$ to $\mu_{15}$ was obtained per weight, in addition to the $\mu_{0}$ (number of atoms), leading to a total of 91 molecular descriptors for each compound. Therefore, the descriptors calculated allowed the adequate codification of the molecular structure.

To obtain the function that allowed to classify, according to characteristic structural related with the RAM, the series of compounds included in the work was developed, by means of the statistical software Statistical 8.5, the Analysis Lineal Discriminant. This technique of analysis multivariate allowed to select, among the ghastly moments included in the womb of data, those with those that the compounds can be classified previously according to its more probable adverse reaction in the established groups, 


\section{Results and Discussion}

Next the 3 canonical equations are shown obtained by means of the analysis discriminant, the equations are shown in hierarchical order, therefore the first one is that of more power discriminant

Table.1 Canonical Equations obtained through the analysis discriminant

\begin{tabular}{|c|c|c|c|}
\hline & Equations 1 & Equations 2 & Equations 3 \\
\hline " $\mu 0 "$ & 0,023101 & 0,055954 & 0,072384 \\
\hline " $\mu$ STD1 & 0,015729 & 0,060715 & 0,064962 \\
\hline " $\mu$ Dip2 & $-0,006297$ & 0,033955 & $-0,069891$ \\
\hline " $\mu \mathrm{Hyd} 2$ & 0,002097 & $-0,046366$ & 0,065878 \\
\hline " $\mu \operatorname{Van} 1$ & 0,028598 & $-0,053820$ & $-0,068237$ \\
\hline $\mu$ Ato1 & 0,029065 & 0,057418 & 0,075964 \\
\hline
\end{tabular}

Table 2 Statisticians of the equations canonical discriminant

\begin{tabular}{|l|l|l|l|l|l|l|}
\hline & Eigen- - value & Canonicl - R & Wilks' - Lambda & Chi-Sqr. & df & p-level \\
\hline $\mathbf{1}$ & 2,052679 & 0,820012 & 0,078227 & 105,7480 & 111 & 0,00622961 \\
\hline $\mathbf{2}$ & 1,131735 & 0,728628 & 0,238801 & 59,4332 & 72 & 0,00855152 \\
\hline 3 & 0,964405 & 0,700671 & 0,509060 & 28,0204 & 35 & 0,00792748 \\
\hline
\end{tabular}

As you it can appreciate in the variables included in the models information so much steric it is coded as electronic of the molecule, fact this that endorses the utility of the obtained models

The Distance of Mahalanobis to the square is a statistician of importance in the analysis discriminant in these cases like that overlapping exists among the groups it can take to the classification of cases in other groups to those that don't belong, next we will discuss these results

For a better understanding of what has thought about show the territorial map based on the graph of the function one versus two 


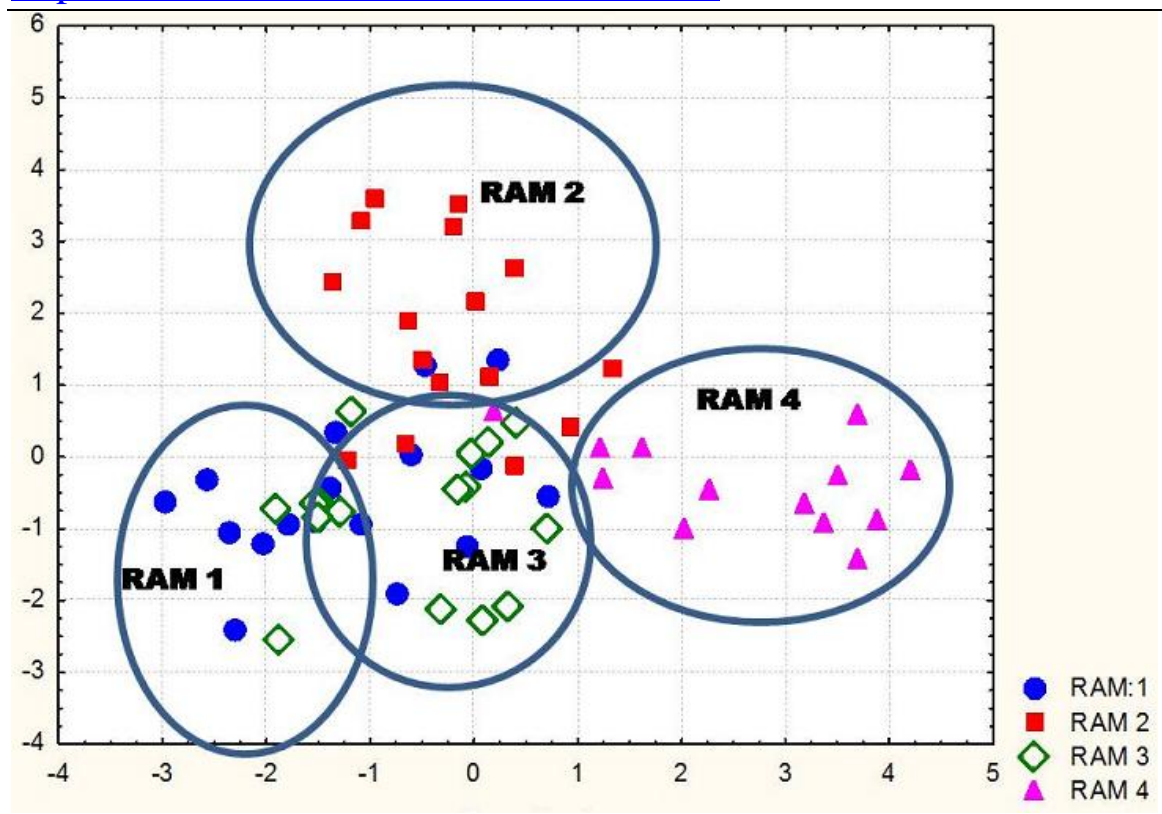

Figure 1. Territorial Map according to classification group

\section{References (}

1. . Ali, H., et al., Mechanisms of inflammation and leukocyte activation. Adv Reumatol, 1997: 1-28.

2. Brune K, Rainsford KD, Schweitzer A. Biodistributión of mild analgesics Br J Clin Pharmacol 1980:279-84.

3. Carda M. Máster en Química Aplicada y Farmacología. Universidad Jaume I. Inflamación: síntesis de antiinflamatorios. 2011.

4. Cramer III, R. D., Patterson, D. E. and Bunce, J. D. J. Am. Chem. Soc., (1988)

5. Crofford LJ. COX-1 and COX-2 tissue expression: implications and predictions. J Rheumatol 1997: 15-9.

6. David E. Golan y col. Principles of Pharmacology: The Pathophysiologic Basic of Drug Therapy, $2^{\text {nd }}$ Edition. Chapter 40: Principles of Inflammation and the Immune System, $p$ 736-74. Chapter 41: Pharmacology of Eicosanoids. p. 748-762,2007.

7. Gerlag, D.M., et al., The effect of a $T$ cell-specific NF-KB inhibitor on in vitro cytokine production and collagen-induced arthritis. J Immunol, 2000: 1652-1658. 
MOL2NET, 2019, 5, ISSN: 2624-5078

http://sciforum.net/conference/mol2net-05

8. Golbraikh A 2002, Tropsha A. Predictive QSAR modeling based on diversity sampling of experimental datasets for the training and test set selection. Molecular Diversity. 2002: $231-243$ 